\title{
Laboratory Investigation on Crack Potential of PG76 Based Stone Mastic Asphalt (SMA) Mixture using a Newly Developed Crack Meander Approach
}

\author{
Mohammed Ayad Al Mashat*, Ratnasamy Muniandy and Salihudin Hassim \\ Master of Engineering, University Putra Malaysia, Malaysia
}

Submission:July 28, 2017; Published: May 02, 2018

*Corresponding author: Mohammed Ayad Al Mashat, Master of Engineering, University Putra Malaysia, Selangor, Serdang, Malaysia, Tel: +00964 7812448 399; Email: mohammedbiap10@gmail.com

\begin{abstract}
With the growth of traffic volume and loading, flexible pavements are confronting an increased growth of fatigue cracking phenomenon worldwide. As an easy and fast simulation of real crack occurrence of bituminous mixtures, crack meander technique using high resolution digital camera (Nikon D5100) was applied in this study. The study involved capturing the crack initiation and propagation on the specimen's surface during the repeated load Indirect Tensile Fatigue Test (ITFT). It was used in order to facilitate the prediction of the remaining fatigue life after the appearance of crack that can be recognized visually by the pavement inspectors. Furthermore, this is to quantify the failure rate by calculating the number of cycles required to cause $1 \mathrm{~mm}$ in length of micro-crack after the cracks propagated. To achieve these objectives, the ITFT raw data namely, the number of cycles to fracture the specimen (Nf), the permanent vertical deformation, and the accumulated permanent vertical strain were used to compare the performance. A newly developed crack meandering approach was used in this study to validate these results. It was concluded that, the crack meander quantification methods agreed with the ITFT results in that moving from coarser aggregate gradation (lower limit) to finer gradation (upper limit) prolonged the fatigue life of SMA significantly.
\end{abstract}

Keywords: Stone mastic asphalt; Crack propagation; Crack meandering; Fatigue life; Maintenance cost

Abbreviations: ITFT: Indirect Tensile Fatigue Test; COPF: Cellulose Oil Palm Fiber; EVA: Ethylene Vinyl Acetate; RSM: Response Surface Methodology; DEM: Discrete Element Method; TSR: Tensile Strength Ratio; MMCM: Measurement and Mapping of the Crack Meander; TDM: Theoretical Maximum Density; UTM: Universal Testing Machine; SMA: Stone Mastic Asphalt

\section{Introduction}

Fatigue crack is a major pattern of structural asphalt pavement distress. Under the action of repeated traffic loading, deterioration of the flexible materials in pavements happened by the accumulation and growth of the micro and macro cracks gradually occur.

As the use of stone mastic asphalt has increased drastically in the last decade, due to its outstanding resistance to permanent deformation, several researchers have examined deferent concepts to evaluate its performance life. One way is by adding different types of fibers (Cellulose Oil Palm Fiber (COPF), and Ethylene Vinyl Acetate (EVA)) with different percentages [1-3], or by reinforcing the asphalt mixtures with waste tire rubber [4]. Other researches indicated improving the fatigue performance by using different types of additives. However, few studies evaluated the effect of changing the aggregate gradation that could affect the fatigue performance.

Therefore, this study is focused on evaluating the effect of aggregate gradation on the fatigue performance of SMA using
ITFT results as well as the newly developed crack meander technique.

\section{Stone Mastic Asphalt and Aggregate Gradation}

Aggregate structure considered as the largest portion of the asphalt mixtures which provides resistance to the applied traffic loads. It is believed that, resistance to repeated load applications is derived by the aggregate strong stone skeleton. Therefore, asphalt mixture performance is directly related to aggregate characteristics particularly the aggregate gradation [5].

Haghshenas HF [6] Have come up with a significant comparison between HMA and SMA stripping potential. Statistical methodology called response surface methodology (RSM) was involved to analyze the results. The effects of aggregate gradation was found to be much higher than asphalt content in increasing the Tensile Strength Ratio (TSR) when each parameter moved from high to mid-level. However, HMA mixtures showed more susceptible to stripping by decreasing the aggregate sizes and increase the asphalt binder content 


\section{Civil Engineering Research Journal}

consequently, while the SMA mixtures under the same conditions showed decrease in moisture resistance.

Yu $\mathrm{H}$ et al. [7] examined the correlation between aggregate gradations and the resulting dynamic modulus. Four aggregate gradation that were involved (one was the basic gradation whose design was obtained based using the superpave mix design procedure, and the other three had gradations varied from the basic gradation by changing the amount of particles passing certain sieve size). This research studied the effect of aggregate size distribution and angularity distribution on dynamic modulus using a 3D discrete element method (DEM). It was understood that the resulting dynamic modulus increased by either increasing $10 \%$ of the particles passing $2.36 \mathrm{~mm}$ and $4.75 \mathrm{~mm}$ sieve, respectively, or reducing the particles passing $9.5 \mathrm{~mm}$ sieve by $10 \%$.

Guler M [8] has investigated the effects of mix design variables (binder type, aggregate gradation, asphalt content, and compaction temperature) on the mechanical properties of HMA based on laboratory compacted specimens using Superpave Gyratory Compactor. It was concluded that the HMA mechanical characteristics during compaction are directly affected by the aggregate gradation and the percent of asphalt binder in a high extent. Results also depicted that binder type is a statistically more valuable parameter than compaction temperature for the mechanical properties.

\section{Fatigue quantification methods}

Few techniques were used to determine the fatigue strength of bituminous mixtures such as the accumulated strain method, dissipated energy method, and visco-elastic continuum damage method. [9] have investigated the fatigue performance of HMA specimens using superpave mix design protocol. It was found that regardless of changing the aggregate gradation, load value or test temperature, the accumulated strain curve continues to increase. In other words, the fatigue life of the mixtures cannot be determined profoundly using this technique.

Widyatmoko et al. [10] studied the fatigue resistance of asphalt mixtures using the energy dissipation approach. Even though this method has an accurate outcomes, it involves complicated analysis as well as the extensive amount of calculation makes it require longer time to draw a conclusion especially with the availability of other simpler and faster techniques in evaluating the fatigue performance.

Lee et al., employed the visco-elastic parameters in order to anticipate the mixture's fatigue life. They found that the fatigue behavior of the bituminous mixtures affected by the viscoelastic characteristics ( $E^{*}$ and $\alpha$ ). Twelve different mixtures combinations have been produced in the laboratory in order to formulate a consistent relationship between the coefficient $\alpha 1$ and $m$ value in the damage determination law where; $\alpha 1=0.5+1 / \mathrm{m}$. Although, this technique was validated by comparing the results of both the real fatigue data and the prediction by applying the equation, choosing a particular coefficient might be not suitable for a specific condition, as well as the statistical percentage of error is risky, which is around $34.4 \%$. Therefore, it is not deniable that this approach does not provide a safe predication in determining the bituminous mixtures fatigue life.

As a conclusion, there is not an obvious, and fast method to evaluate the fatigue performance of the bituminous mixtures nor a specific standard that defines the best approach to examine the performance among the various asphalt mixtures combinations. Hence, evaluating the fatigue crack initiation and migration as well as its pattern is required in order to portrait the crack development concept.

\section{Crack Meander Concept}

The term "crack meandering" was derived from the concept of a stream with a convoluted path by Muniandy. Various definitions have been stated to elaborate the definite meaning of this approach. According to the Oxford definition, meander means 'to curve a lot rather than being in a straight line' or 'to walk slowly and change direction often, especially without a particular aim'. To wrap this up, meander is the migration of the cracks on the sample surface due to the influence of the repeated load operations.

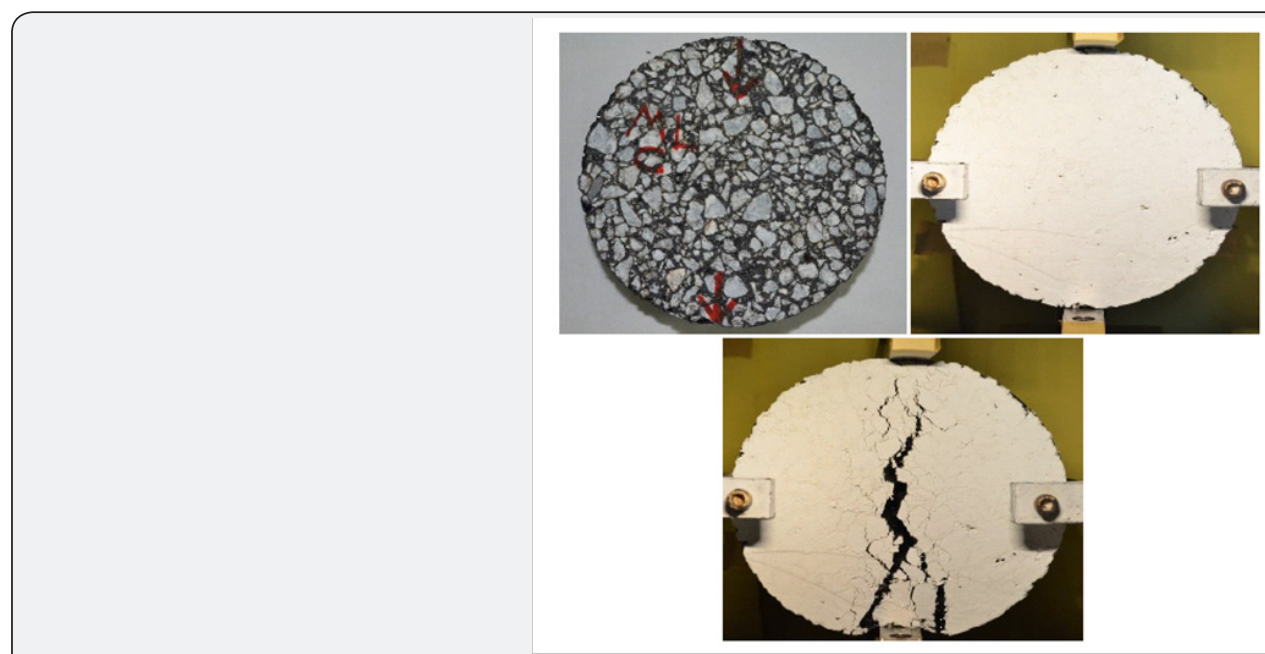

Figure 1: Trimmed Sample before and after Painting and at failure. 
The crack meander study is mainly based on the observation of the crack appearing on a sample surface as the ITFT is taking place. Furthermore, some modification on the holding frame of the Universal Testing Machine is required to facilitate capturing the whole surface of the sample, as shown in Figure 1 below. A high resolution camera, with the ability of taking multiple shots per second was used so that best pictures will be picked for the analysis stage. Each picture was coded by using cycles around.

Few studies on crack meandering after its introduction in 2003 have been conducted in order to develop this approach effectively. Sirous R, developed the crack meander protocol for fatigue resistance of SMA as well as developed measurement and mapping of the Crack Meander software (MMCM) which is powerful in determining crack length, and area after digitizing the sample images using online image editor software as presented in Figure 2. Eventually, Muniandy et al. [2] studied the effect of using different types of fibers namely cellulose oil palm fiber (COPF), and ethylene vinyl acetate (EVA) on the fatigue strength of SMA using ITFT and validated the results by crack meander approach. They concluded that there is a correlation between crack meandering approach and ITFT data.

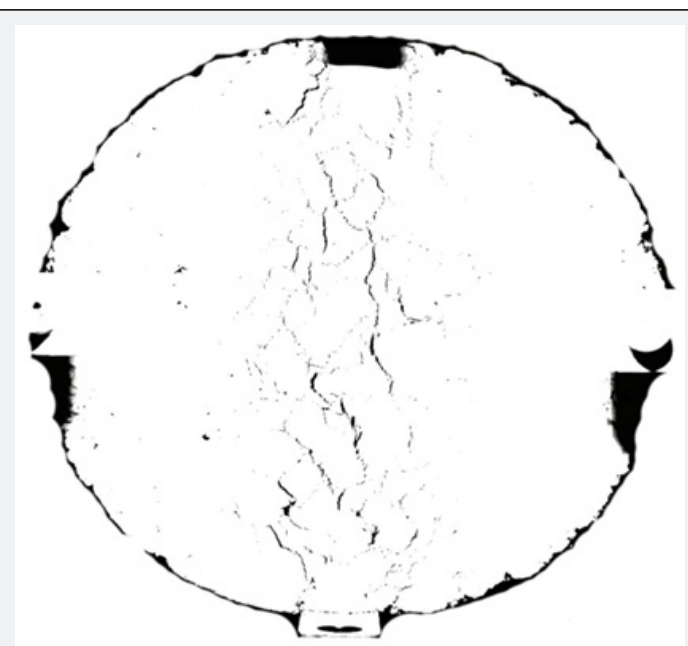

Figure 2: Schematic Image used for crack migration rate calculation.

\section{Research Approach}

For the purposes of this study, superpave mix design was used in the laboratory to fabricate eight consistent SMA specimens ( $100 \mathrm{~mm}$ diameter) for each of 3 aggregate gradation matrixes in order to determine the optimum binder content. Where two samples have been prepared for each asphalt content namely; 5.0, 5.5, 6.0, 6.5\%, as well as the Theoretical Maximum Density (TMD) samples. Consequently, nine more specimens were prepared using the optimum asphalt content to conduct ITFT, three samples for each aggregate gradation. The ITFT was performed on samples with $150 \mathrm{~mm}$ diameter to determine the change in fatigue life as function of aggregate gradation change. In addition to that, the crack initiation and propagation on the surface of the sample during the fatigue test was observedto validate the results obtained from the indirect tensile fatigue test.

\section{Materials Used}

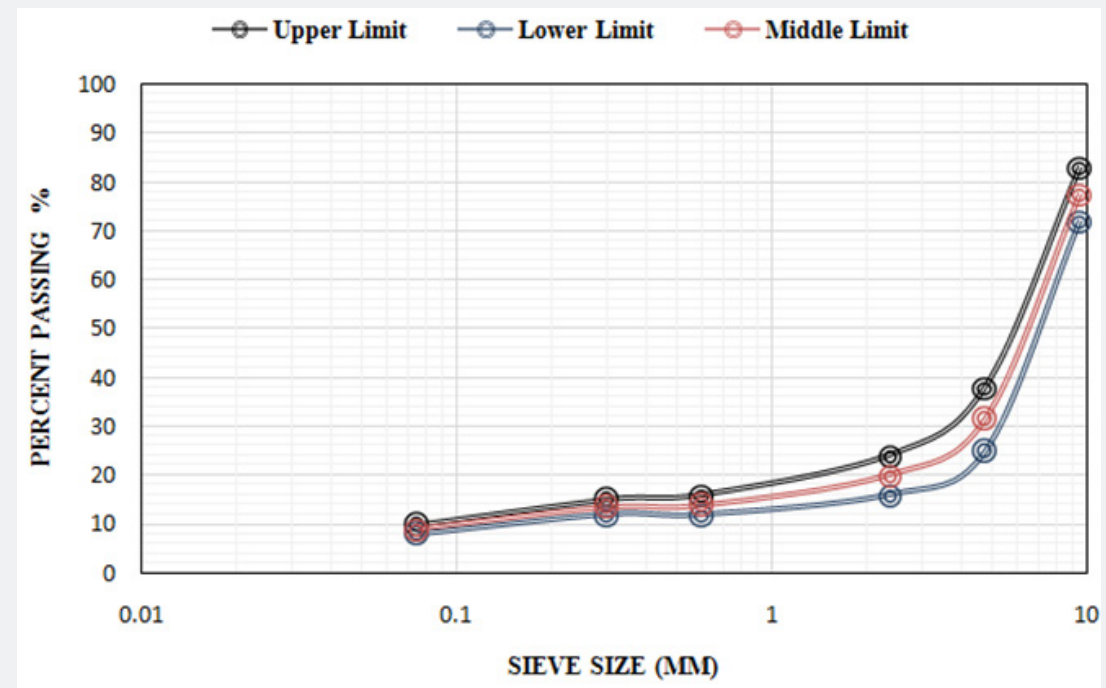

Figure 3: Aggregate gradation curves for, Lower, Middle, Upper limit. 


\section{Civil Engineering Research Journal}

Table 1: Properties of Coarse and Fine aggregate.

\begin{tabular}{|c|c|c|c|}
\hline Properties & Result & Specification & Standards \\
\hline L.A abrasion & 17.8 & $<30 \%$ & ASTM C 131 \\
\hline Flakiness Index & 9.95 & $<25 \%$ & ASTM C 4791 \\
\hline Elongation Index & 11.2 & $<25 \%$ & ASTM D 4791 \\
\hline Aggregate Impact Value & $9.90 \%$ & $15 \%$ & BS 812 Part III \\
\hline \multicolumn{4}{|c|}{ Coarse Angularity } \\
\hline \multicolumn{4}{|c|}{ One Fracture Face } \\
\hline $9.5 \mathrm{~mm}$ & $95 \%$ & $95 \%$ & ASTM D 5821 \\
\hline $4.75 \mathrm{~mm}$ & $97 \%$ & $95 \%$ & ASTM D 5821 \\
\hline \multicolumn{4}{|c|}{ Two or more fractured face } \\
\hline $9.5 \mathrm{~mm}$ & $88 \%$ & $90 \%$ & ASTM D 5821 \\
\hline $4.75 \mathrm{~mm}$ & $92 \%$ & $90 \%$ & ASTM D 5821 \\
\hline \multicolumn{4}{|c|}{ Fine Aggregate } \\
\hline $0.6 \mathrm{~mm}$ & $51 \%$ & $45 \%$ & AASHTO T304 \\
\hline $0.3 \mathrm{~mm}$ & $46 \%$ & $45 \%$ & AASHTO T304 \\
\hline
\end{tabular}

Table 2: Properties of binder PG76.

\begin{tabular}{|c|c|c|c|}
\hline Properties & Result & Specification & Standards \\
\hline Penetration & $29.7(0.1 \mathrm{~mm})$ & -- & ASTM 5 \\
\hline Softening Point & $81.85^{\circ} \mathrm{C}$ & $>60^{\circ} \mathrm{C}$ & ASTM D36 \\
\hline Flash and Fire Point & & & ASTM D92 \\
\hline Flash Temperature & $230^{\circ} \mathrm{C}$ & $230^{\circ} \mathrm{C}$ & ASTM D92 \\
\hline Fire Point & $275^{\circ} \mathrm{C}$ & -- & ASTM D 4402 \\
\hline Viscosity & & & ASTM D 4402 \\
\hline At $135^{\circ} \mathrm{C}$ (centipoise) & 1543.9 & --- & - \\
\hline At $165{ }^{\circ} \mathrm{C}$ & 433.8 & - & \\
\hline
\end{tabular}

Figure 3 below demonstrates the different gradation distributions of SMA-14 namely, lower, middle, and upper limits which are used in this study with nominal maximum aggregate size of $9.5 \mathrm{~mm}$ according to the Malaysian standards [JKR/ SPJ/2008]. PG76 asphalt binder was employed in this study

\section{Specimen Preparation}

Table 3: Summary of the volumetric properties.

\begin{tabular}{|c|c|c|c|c|c|c|c|c|c|c|c|}
\hline $\begin{array}{l}\text { Gradation } \\
\text { Limit }\end{array}$ & OAC $\%$ & $\begin{array}{l}\text { \%Gmm } \\
\text { @Nini }\end{array}$ & Specs. & $\begin{array}{l}\text { \%Gmm } \\
@ N m a x\end{array}$ & Specs. & $\begin{array}{c}\text { Air } \\
\text { Voids \% }\end{array}$ & VMA & Specs. & $\begin{array}{c}\text { VCA mix } \\
\%\end{array}$ & $\begin{array}{c}\text { VCA } \\
\text { Dry \% }\end{array}$ & Specs. \\
\hline Lower & 6.9 & 88.6 & \multirow{3}{*}{$<89 \%$} & 97.2 & \multirow{3}{*}{$<98 \%$} & 4 & 18.5 & \multirow{3}{*}{$>17 \%$} & 27.6 & 40.41 & \multirow{3}{*}{ VCAmix $<$ VCAdry } \\
\hline Middle & 6.5 & 87.3 & & 97.5 & & 4 & 17.56 & & 29.9 & 41.15 & \\
\hline Upper & 5.7 & 87.5 & & 97.6 & & 4 & 16.08 & & 32.8 & 41.88 & \\
\hline
\end{tabular}

Superpave mix design protocol was employed to produce three different mixtures using three different aggregate gradation limits for SMA-14. After the samples had cold down, it is trimmed from both sides to achieve the desired height as $60 \mathrm{~mm}$ using the trimming machine. Table 3 presents the final results of the mixtures properties. One side of the sample surface was painted using water base white color paint prior to conducting the ITFT test in order to visualize the crack initiation, propagation, and as well which is recommended in the Malaysian standards for SMA. The characteristics of granite aggregate and asphalt binder are shown in Table $1 \& 2$. Furthermore, the limestone filler was added to the mixture in addition to the $0.3 \%$ cellulose oil palm fiber by weight of the total aggregate. 


\section{Civil Engineering Research Journal}

\section{Indirect Tensile Fatigue Test (ITFT)}

This test was performed using the Universal Testing machine (UTM) at UPM Highway Laboratory and the number of cycles to failure, total permanent vertical deformation, and accumulated permanent vertical strain were obtained as raw data and their graphs produced by UTSO13 software. For the total permanent vertical deformation, (N2) point was specified as the comparison benchmark which is the point where the deformation slope becomes bias to the vertical axis prior to failure as shown in Figure 5. ratio will be employed in this study as one of the quantification methods for ITFT which means the change in accumulated permanent vertical strain in respect to the number of cycles all over the fatigue life of the specimens. It will be performed by dividing the fatigue life for each aggregate gradation into six regions in order to determine the vertical strain induction rate all over the fatigue life of the sample which may give an indication of the real behavior of the pavement and the proper rehabilitation required for that situation is presented in Table 4. The sample is considered failed when the displacement reached $10 \mathrm{~mm}$ where this is the maximum LVDT sensor limit. In this experiment, $150 \mathrm{~mm}$ in diameter samples were used with a thickness of approximately $60 \mathrm{~mm}$. Table 5 illustrates the setting parameters employed in ITFT.

Table 4: Measured crack length and area.

\begin{tabular}{|c|c|c|c|}
\hline \multicolumn{3}{|c|}{ Ave. N.C/L } & 419.8 \\
\hline No. of Cycles & Area $\left(\mathbf{m m}^{\mathbf{2}}\right.$ ) & Length $\mathbf{( m m )}$ & $\begin{array}{c}\text { N.C/L (Cycles/ } \\
\mathbf{m m})\end{array}$ \\
\hline 88,000 & 72 & 36 & 2444.4 \\
\hline 89,250 & 250 & 50 & 1785 \\
\hline 89,300 & 300 & 60 & 1488.3 \\
\hline 89,371 & 420 & 60 & 1489.5 \\
\hline \multicolumn{4}{|c|}{ Ave. N.C/L } \\
\hline
\end{tabular}

Table 5: Test parameters for the repeated ITFT.

\begin{tabular}{|c|c|}
\hline Test Parameters & Value \\
\hline Seating force & $100 \mathrm{~N}$ \\
\hline Cycle loading force & $3500 \mathrm{~N}$ \\
\hline Loading duration & $100 \mathrm{~ms}$ \\
\hline Cycle repetition time & $500 \mathrm{~ms}$ \\
\hline Poisson ratio & 0.35 \\
\hline
\end{tabular}

As recommended in the British standards (BS DD123) and since $1 \mathrm{~Hz}$ is time consuming, the load frequency was fixed to be $2 \mathrm{~Hz}$ which means the cycle duration will be 0.5 second; $0.1-0.4 \mathrm{~s}$ (100-400ms). Poisson's ratio of 0.35 was specified as was recommended for asphalt mixtures [11]. The load value was $3500 \mathrm{~N}$. Temperature of $20^{\circ} \mathrm{C}$ is used as it is a worldwide employed temperature for fatigue resistance tests. Therefore, the samples were required to be conditioning for at least two hours to reach the desired testing temperature. The modified frame was used rather than the original one so that the full specimen's diameter surface can be captured completely as presented in Figure 2.

\section{Crack Meander Technique}

Besides evaluating the fatigue life of SMA as a function of aggregate gradation, this study was aimed to validate the ITFT data by using the crack meander technique. High resolution digital camera (Nikon D5100) was employed to perform this validation experiment, as the distance and height of the camera with respect to the sample inside the UTM were fixed the same for all samples by using a stable tripod as well as capturing the images conducted smoothly through the class door of the machine during the ITFT [12].

Two quantification methods were conducted in this technique to correlate the fatigue strength in terms of 3 different gradation matrixes. First of all, predicting the percentage of remaining fatigue life after the macro-crack appears on the sample surface which was done by comparing the crack initiation and specimen failure during ITFT for all samples. Crack initiation point was chosen in this study as it is an easy way for the highway inspectors to notice during the pavement condition evaluation where they should report to start the rehabilitation work prior to any serious damages happen. Secondly, the number of cycles required to cause $1 \mathrm{~mm}$ of crack length after the cracks propagated. After the macro-crack appears on the surface, its propagation rate will accelerate drastically, therefore this quantification approach has been studied to give proper warning signs regarding the damaging precipitation. Figure 3 presents the samples of the digitized image which clearly shows the micro and macro cracks initiated, propagated and migrated horizontally and vertically on the applied load axis. Image editor software was used to digitize the images of the samples, which were used to calculate the macro- crack initiation and migration rate in respect of different number of cycles and aggregate gradation. The migration rate was manually calculated to determine the number of repeated cycles required to cause $1 \mathrm{~mm}$ of macro-crack length. Using the same scale for all images in order to calculate the length of the cracks during the fatigue test and the average number was taken for each aggregate gradation for the comparison purposes [13].

\section{Results and Discussion}

Quantification methods of fatigue strength using two approaches were compared. The first one was done by ITFT, and the second one by crack meander approach. Three methods were based on the ITFT raw results which are namely; the number of cycles to failure, total permanent vertical deformation, and accumulated permanent vertical strain. Two analysis methods depended on the crack meander quantification technique; the percentage of remaining life after the macro-crack appears on the sample surface and number of cycles required to cause $1 \mathrm{~mm}$ of crack length, which were validated by comparing them with the ITFT raw data. The summary of macro crack area, length and migration length is as shown in Table 6. 


\section{Civil Engineering Research Journal}

Table 6: Macro-cracks Area, Length, and migration calculation.

\begin{tabular}{|c|c|c|c|}
\hline \multicolumn{4}{|c|}{ Lower Limit } \\
\hline No. of Cycles & Area $\left(\mathrm{mm}^{2}\right)$ & Length (mm) & N.C/L (Cycles/mm) \\
\hline 12,250 & 60 & 35 & 350 \\
\hline 12,780 & 120 & 40 & 319.5 \\
\hline 12,880 & 160 & 46 & 280 \\
\hline 13,150 & 180 & 50 & 263 \\
\hline 13,220 & 165 & 55 & 240.4 \\
\hline 13,260 & 180 & 60 & 221 \\
\hline Ave. N.C/L & 279 & & \\
\hline \multicolumn{4}{|c|}{ Middle Limit } \\
\hline No. of Cycles & Area $\left(\mathrm{mm}^{2}\right)$ & Length (mm) & N.C/L (Cycles/mm) \\
\hline 22,600 & 144 & 48 & 470.8 \\
\hline 22,850 & 180 & 52 & 439.4 \\
\hline 23,000 & 224 & 56 & 410.7 \\
\hline 23,050 & 308 & 56 & 411.6 \\
\hline 23,080 & 390 & 63 & 366.3 \\
\hline
\end{tabular}

\section{Indirect Tensile Fatigue Test}

ITFT was performed using control stress loading mode in compliance with the BS DD 213 standard. In this study, three

Number of Cycles Vs Aggregate Gradations different quantification approaches were based on the raw data of ITFT namely; number of cycles to failure, total permanent vertical deformation, and accumulated permanent vertical strain, with respect to changing the aggregate gradation matrix.

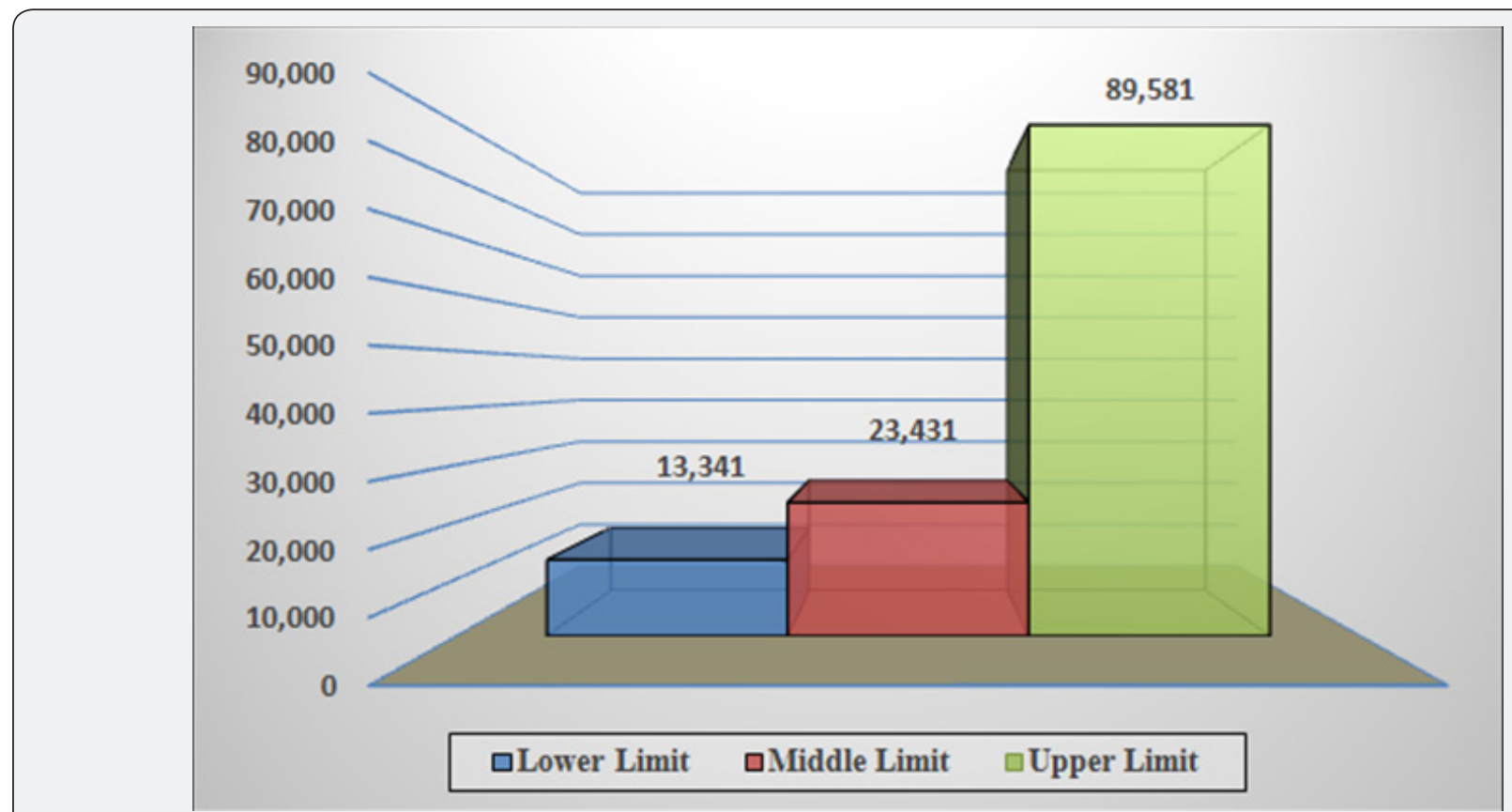

Figure 4: N.C till failure Vs. Aggregate Gradation.

The first indication of the mixture fatigue performance is the number of load repetitions until the sample fails. The failure definition in this case is the sample fracture which occurs when the vertical deformation reaches $10 \mathrm{~mm}$. Figure 4 below demonstrates the number of load cycles to fracture the sample for each aggregate gradation combinations. The results show that the upper limit has the longest fatigue life in comparison with the other two gradation matrixes [14]
Total Permanent Vertical Deformation Vs Aggregate Gradation

Figure 5 below shows the location of the crack propagation critical point when the vertical deformation gradient changes drastically and becomes bias to vertical axis just before the fracture point as a basis of the comparison for all gradation combinations. This point can be considered as a critical point to perform the partial or full-depth pavement rehabilitation works. 


\section{Civil Engineering Research Journal}

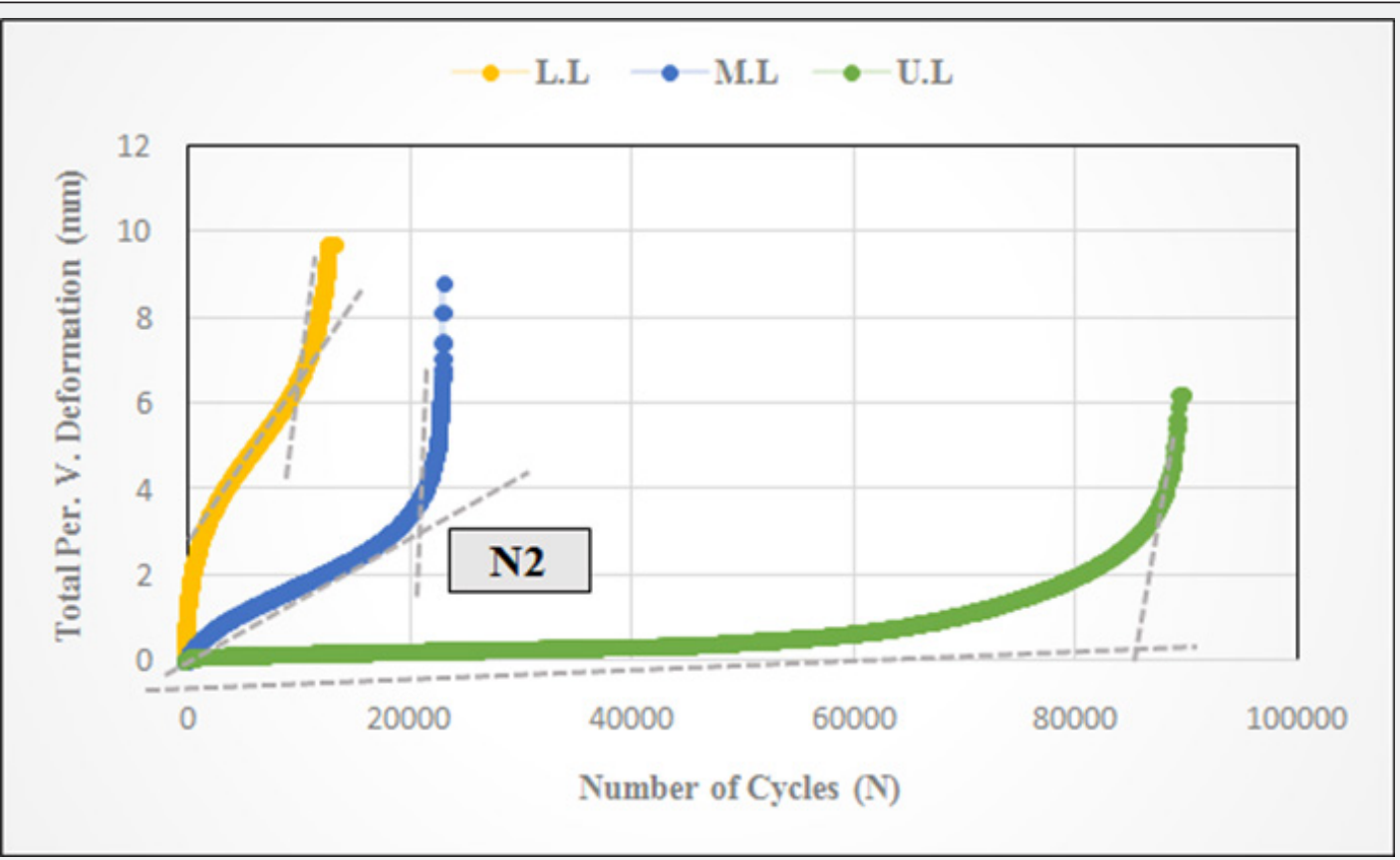

Figure 5: Total Permanent Vertical Deformation Compression.

Furthermore, it presents the comparison among the three aggregate matrixes in terms of the total permanent vertical deformation at N2, which shows, that the finer gradation (upper limit) develops far higher strength to the total permanent vertical deformation prior to its failure equal to only $0.6 \mathrm{~mm}$ while the coarser limit develops $7.5 \mathrm{~mm}$ of vertical deformation at the same point. Therefore, the finer limit can be ranked as the best aggregate combination for providing the highest vertical deformation resistance [15].

\section{Accumulated permanent vertical strain Vs aggregate gradation}

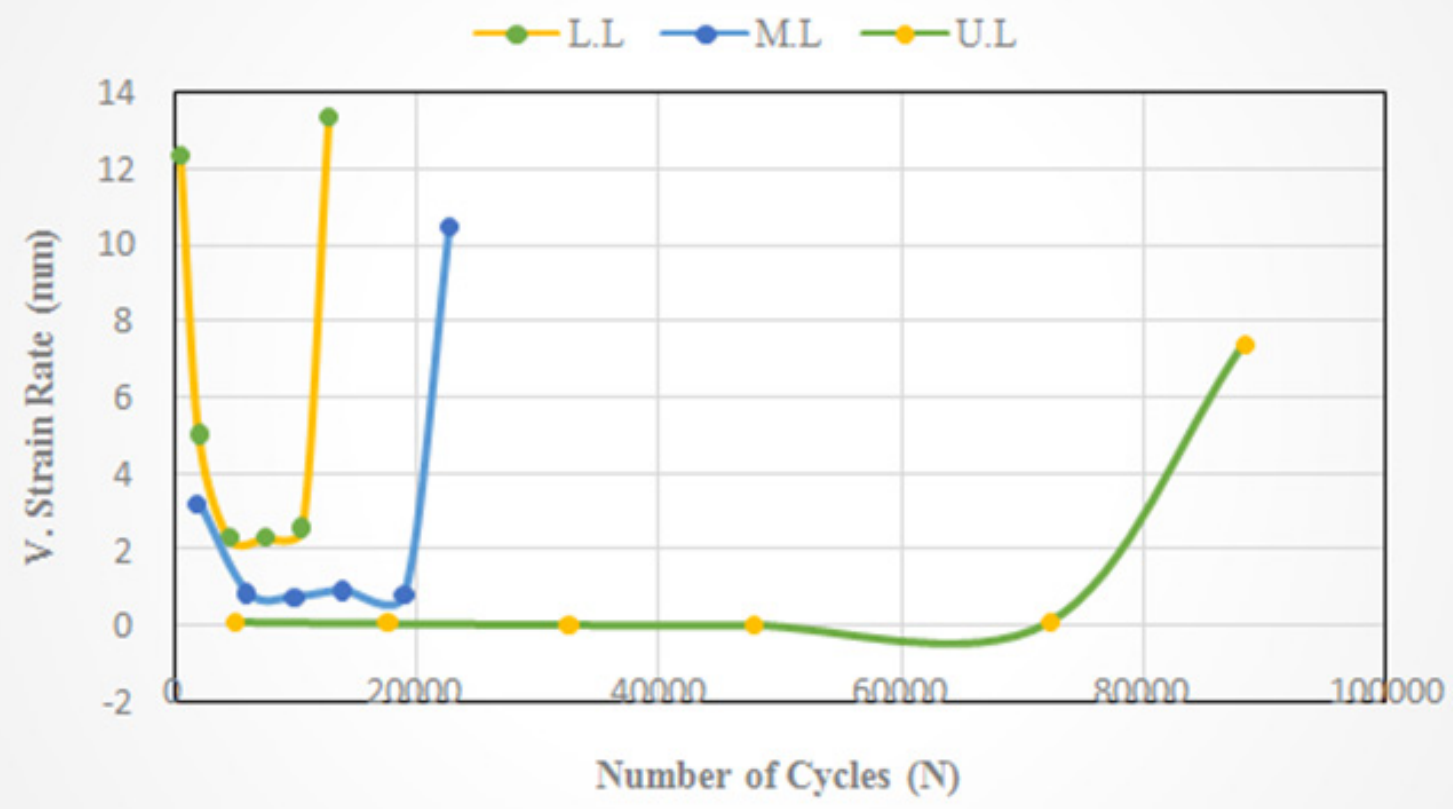

Figure 6: Total Permanent Vertical Deformation Compression.

The rate of change of the vertical strain with respect with number of cycles is employed in this section as ratio, to demonstrate how the compression strain develops during the life of the sample under specific conditions of load and temperature. Figure 6 illustrates the vertical strain rate vs number of cycles to failure. The strain induced in upper limit has the lowest development rate. Therefore, it performs as the best aggregate gradation limit in terms of resisting the fatigue cracking for SMA-14 mixtures due to its denser nature. 


\section{Civil Engineering Research Journal}

\section{Crack Meander Technique}

Crack meandering method was involved in this study to analysis the images that have been captured during the ITFT for computing the remaining life after the cracks appear on the sample surface, and to evaluate the fatigue strength after the cracks propagated.

Percentage of Fatigue Life Prior and after Crack Appears

The crack initiation was captured by the high resolution camera during ITFT in order to observe and record the crack initiation, propagation and migration of the mixtures samples. The pictures of all aggregate gradations were used to calculate the percentage of the remaining life before and after the crack appears and until the failure. This is as shown in Figure 7 below. The results indicate that the upper aggregate gradation limit has the longest fatigue life until the cracks appear, which is $17.9 \%$ of the whole fatigue life $(16,034$ cycles), as well as the longest life after the crack appears until the failure $(73,547$ cycles). The cracks appear-point determination could help the road authorities in scheduling and predicting the maintenance stage before the distresses are further aggravated and more allocations are required.

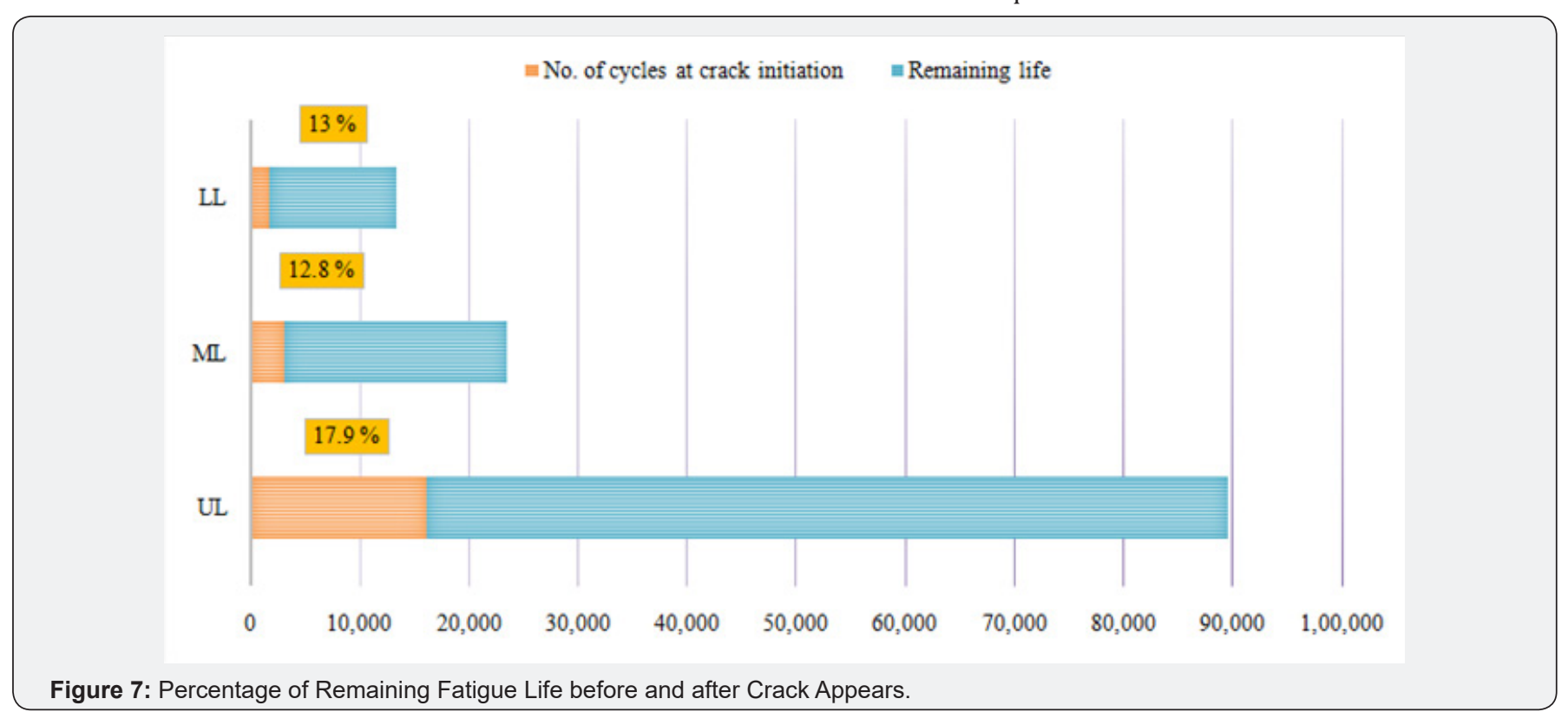

Number of Cycles to Cause $(1 \mathrm{~mm})$ of Macro-Crack

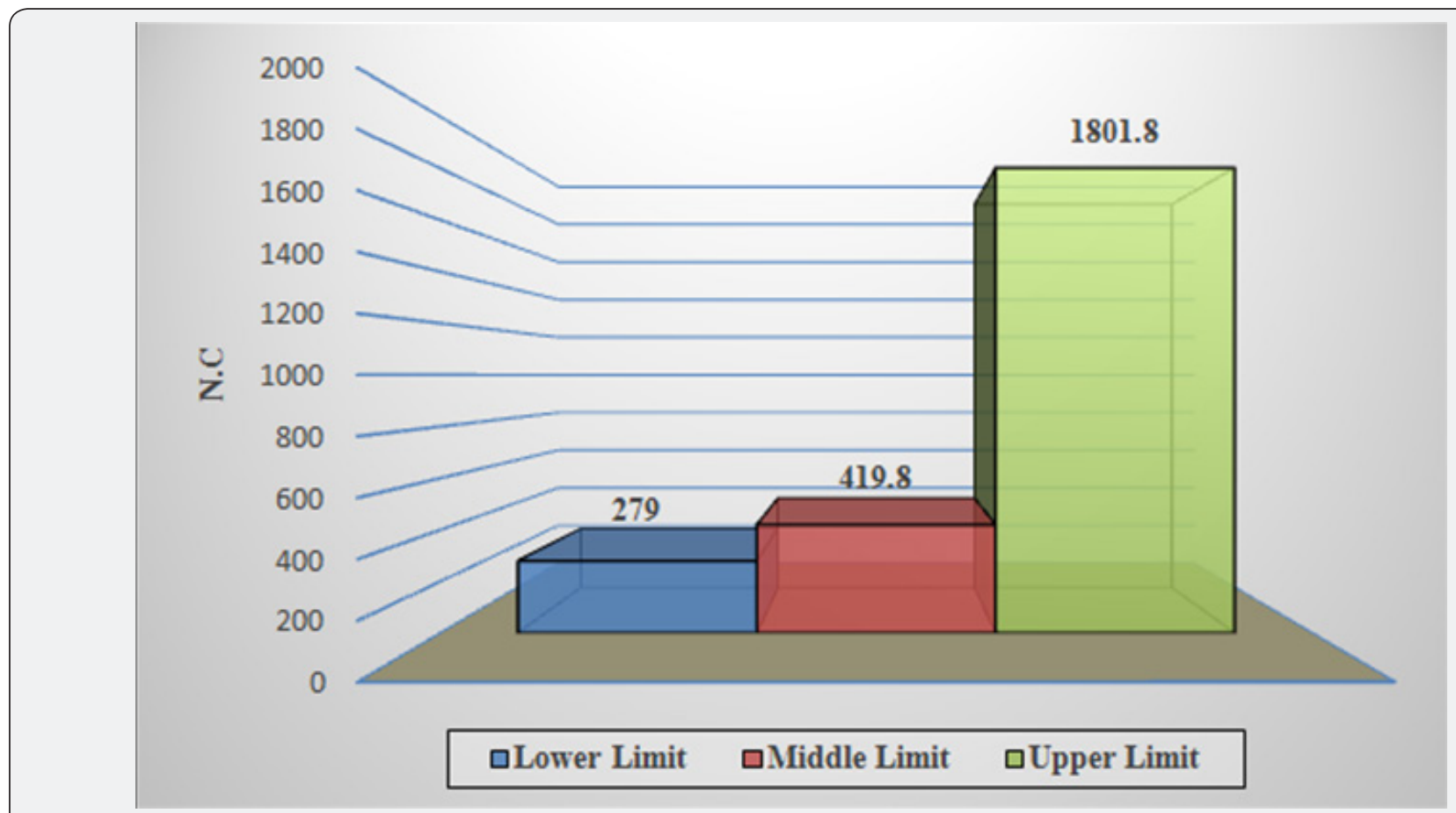

Figure 8: N.C to Cause $(1 \mathrm{~mm})$ of macro-crack. 


\section{Civil Engineering Research Journal}

Table 5 shows the tabulation of the area, length of macrocracks appeared on the surface of the SMA mixtures. By selecting specific images, the number of cycles required to cause one $\mathrm{mm}$ of macro-cracks for that images were recorded and calculated. Finally, the average number of cycles for each aggregate gradation was determined in used for the comparison purposes. Figure 8 demonstrates the comparison between the three aggregate combinations involved in this study, which indicated that the upper limit presents better performance which is required about 1802 cycles in average to cause $1 \mathrm{~mm}$ of macro cracks appeared on the sample surface during ITFT, while the coarsest limit (lower limit) needed only 279 cycles in average to make $1 \mathrm{~mm}$ of macro cracks.

\section{Conclusion}

The main objective of this study was to evaluate the effect of changing the aggregate matrixes on the Stone Matrix Asphalt (SMA) fatigue performance using the Indirect Tensile Fatigue Test on cylindrical specimens of $150 \mathrm{~mm}$ diameter. Prior to conducting the ITFT, the first stage of this study included, the physical properties of the aggregates, and asphalt binder has been evaluated to make sure they are within the global, and Malaysian road specifications. Furthermore, finding out the correlation between cracks appearance on the sample surface with the remaining fatigue life.

The three aggregate gradation combinations namely, lower, middle, and upper limits were selected based on the Malaysian standards (Public Work Department, Malaysia, 2000). The Coarse/Fine percentages were varied to predict the fatigue performance accordingly. The lower limit has the highest $\mathrm{C} / \mathrm{F}$ percentage (contains the highest coarse percentage), while the upper limit contains of the lowest $\mathrm{C} / \mathrm{F}$ percentage.

Based on the results of the fatigue performance test, it can be concluded that:

a. The upper limit combinations have higher number of cycles to failure, and lower permanent vertical deformation.

b. By observing the crack initiation on the sample surface during the ITFT, the fatigue life to cracks appearance, as well as the fatigue life from cracks appearance till the failure stage could be determined. Again the finer limit indicated the longest fatigue life before and after the macro-crack appears.

c. In terms of evaluating the fatigue strength after the cracks propagated, as the coarse/fine percentages getting lower, the performance progressively and drastically enhanced as the interconnection increases due to the existence of the fine aggregate.

d. Finally, the fatigue resistance could be increased by moving from the coarse combinations to the fine combinations. Therefore, employing the upper limit matrix for the SMA-14 for the Malaysian specifications, will lead to better fatigue performance and less allocations for maintenance accordingly.

e. These outcomes come harmonious with the literature review (Nejad et al., 2010) which stated that HMA mixtures perform better in terms of fatigue behavior than SMA mixtures using the same nominal maximum aggregate size.

\section{Recommendations}

Since few conclusions have been drawn from this study some recommendations are given future investigations, as summarized below:

a. In order to achieve a statically significant confidence in predicting the fatigue life based on the crack meander technique, further studies using various asphalt mixtures combinations properties, as well as changing the ITFT parameters are required.

b. Studying the effect of the aggregate gradations on the SMA fatigue performance, it is better to use different types of aggregate, aggregate gradation, different mixing methods, and different compaction methods.

c. Using confined test techniques in evaluating the fatigue behavior of asphalt mixtures in the laboratory as better simulation of the field.

\section{References}

1. Muniandy R, Huat BB (2006) Laboratory diameteral fatigue performance of stone matrix asphalt with cellulose oil palm fiber. American Journal of Applied Sciences 3(9): 2005-2010.

2. Muniandy R, Akhir NABCM, Hassim S, Moazami D (2014) Laboratory fatigue evaluation of modified and unmodified asphalt binders in stone mastic asphalt mixtures using a newly developed crack meander technique. International Journal of Fatigue 59: 1-8.

3. Sirous Radkeya (2011) Development of crack meander protocol for the fatigue resistance of stone mastic asphalt mixture using cellulose fibers. PhD thesis, University Putra Malaysia, Malaysia.

4. Mashaan NS, Ali AH, Koting S, Karim MR (2013) Dynamic properties and fatigue life of stone mastic asphalt mixtures reinforced with waste tyre rubber. Advances in Materials Science and Engineering.

5. Roberts FL, Kandhal PS, Brown ER, Lee DY, Kennedy TW (1996) Hot mix asphalt materials, mixture design and construction?

6. Haghshenas HF, khodaii A, hossain M, gedafa DS (2015) Stripping potential of HMA and SMA: a study using statistical approach. Journal of materials in civil engineering 27(11).

7. Yu H, shen S (2012) Impact of aggregate packing on dynamic modulus of hot mix asphalt mixtures using three-dimensional discrete element method. Construction and building materials 26(1): 302-309.

8. Guler M (2008) Effects of mix design variables on mechanical properties of hot mix asphalt. Journal of transportation engineering 134(3): 128-136.

9. Abo-Qudais S, Al-Shweily H (2007) Effect of aggregate properties on asphalt mixtures stripping and creep behavior. Construction and building materials 21(9): 1886-1898.

10. Widyatmoko I, Ellis C, Read JM (1999) The application of the dissipated energy method for assessing the performance of polymer-modified bituminous mixtures. Materials and Structures 32(4): 304-310. 


\section{Civil Engineering Research Journal}

11. Huang YH (1993) Pavement analysis and design.

12. Jabatankerjaraya, Public works department (2000) Standard specification for road works, road branch, public works department, Kula Lumpur

13. Nejad FM, Aflaki E, Mohammad IMA (2010) Fatigue behavior of SMA and HMA mixtures. Construction and building materials 24(7): 1158 1165.

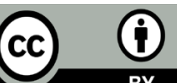

This work is licensed under Creative Commons Attribution 4.0 License

DOI: 10.19080/CERJ.2018.04.555647
14. Lee HJ, Kim YR (1998) Viscoelastic constitutive model for asphalt concrete under cyclic loading. Journal of Engineering Mechanics 124(1): 32-40.

15. Castro M, Sánchez JA (2008) Estimation of asphalt concrete fatigue curves-a damage theory approach. Construction and building materials 22(6): 1232-1238.

\section{Your next submission with Juniper Publishers} will reach you the below assets

- Quality Editorial service

- Swift Peer Review

- Reprints availability

- E-prints Service

- Manuscript Podcast for convenient understanding

- Global attainment for your research

- Manuscript accessibility in different formats

( Pdf, E-pub, Full Text, Audio)

- Unceasing customer service

Track the below URL for one-step submission https://juniperpublishers.com/online-submission.php 